\title{
Magnesium Fluoride Whispering Gallery Mode Disk-Resonators for Microwave Photonics Applications
}

\author{
Hervé Tavernier, Patrice Salzenstein, Kirill Volyanskiy, Yanne Kouomou Chembo, and Laurent Larger
}

\begin{abstract}
We have manufactured a high- $Q$ whispering gallery mode resonator with magnesium fluoride for microwave photonics applications in the gigahertz frequency range. This crystal is scarcely used for resonator fabrication despite its numerous advantages, that are mainly high mechanical hardness, low sensitivity to water vapor pollution, and low sensitivity to photo- and thermo-refractive fluctuations at optimal temperatures. Using a customized machining procedure, we have successfully fabricated and characterized a 5-mm resonator with a surface rugosity of the order of $1 \mathrm{~nm}$ (from 3 to 12 atoms). Cavity ring-down measurements enabled us to determine that the resonator has a quality factor $Q=3.4 \times 10^{8}$ at $1550 \mathrm{~nm}$.
\end{abstract}

Index Terms-Magnesium fluoride, optical cavity, whispering gallery mode (WGM) resonator.

W HISPERING gallery mode (WGM) optical resonators have been the focus of an increasing amount of scientific research in recent years. This interest has been driven by the essential advantages of monolithic WGM technology: low-cost, conceptual simplicity, and low energetic losses (see [1] and references therein). WGM resonators are also particularly interesting because they are central components for a wide variety of applications in optics and in microwave photonics. In the linear regime, for example, WGM resonators can be used as extremely narrow optical filters [2], [3], useful in optical communications or in optoelectronic oscillators [4]. In the nonlinear regime, they can enable optical frequency comb generation [5], [6], erbium-ions-induced lasing [7], Raman lasing [8], or Brillouin lasing [9]. From a broader perspective, WGM resonators are expected to play a key role in time-frequency metrology, integrated photonic circuits, sensing, and aerospace engineering.

So far, in microwave photonics applications, passive millimeter-size WGM resonators have mainly been manufactured with fused silica, quartz, or calcium fluoride $\left(\mathrm{CaF}_{2}\right)$. On the one hand, fused silica is an amorphous medium that is nowadays a standard transparent material at $1550 \mathrm{~nm}$, owing the optical

Manuscript received June 19, 2010; revised July 23, 2010; accepted August 15, 2010. Date of publication September 13, 2010; date of current version October 22, 2010. This work was supported by the ANR research project O2E, and by the Centre National d'Etudes Spatiales (CNES).

H. Tavernier, K. Volyanskiy, Y. K. Chembo, and L. Larger are with the Optics Department, FEMTO-ST Institute (UMR CNRS 6174), 25030 Besançcon Cedex, France.

P. Salzenstein is with the Time-Frequency Department, FEMTO-ST Institute (UMR CNRS 6174), 25030 Besançcon Cedex, France.

Color versions of one or more of the figures in this letter are available online at http://ieeexplore.iee.org.

Digital Object Identifier 10.1109/LPT.2010.2075923 fiber communications applications. The related manufacturing processes enabled the fabrication of WGM resonators with $Q=8 \times 10^{9}$ at $633 \mathrm{~nm}$ (very near the ultimate theoretical limit, see [10]), where $Q$ is the ratio between the central frequency and the bandwidth of the resonance. It can, therefore, be deduced by inference that $Q$-factors superior to $10^{9}$ can in principle be obtained at $1550 \mathrm{~nm}$. On the other hand, crystalline media generally enable to achieve superior performances. For example, quartz WGM resonators with a $Q$-factor of $5 \times 10^{9}$ at $1550 \mathrm{~nm}$ have already been reported [11]. However, $\mathrm{CaF}_{2}$ seems to be the most interesting alternative as it yields the highest $Q$-factor reported so far at $1550 \mathrm{~nm}$, a record value of $1.1 \times 10^{10}[12]$.

In this letter, we consider an unusual bulk medium for the resonator, magnesium fluoride $\left(\mathrm{MgF}_{2}\right)$. This tetragonal crystal has many specific advantages that may be of particular interest for various applications. For example, $\mathrm{MgF}_{2}$ is not sensitive to water vapor pollution [13], [14]. Its lifetime in free atmosphere is, therefore, significantly longer that the one of fused silica, which generally needs a purged nitrogen local atmosphere, or a special surface treatment. Another advantage of $\mathrm{MgF}_{2}$ is its mechanical hardness, which is in the range of 6 Mohs. This relatively high value makes this material resistant to mechanical shocks and to hazardous surface scratches, at the opposite of $\mathrm{CaF}_{2}$ which is mechanically softer. Therefore, $\mathrm{MgF}_{2}$ resonators are relatively easy to manipulate and their $Q$-factors are satisfyingly stable over time. Table I presents a comparison of some interesting properties of fused silica, quartz, $\mathrm{CaF}_{2}$ and $\mathrm{MgF}_{2}$ materials. The advantageous properties of $\mathrm{MgF}_{2}$ appear straightforwardly: it is the unique material having at the same time a good resistance to mechanical shocks and a high resistance to water pollution.

Magnesium fluoride WGM resonators also have other positive characteristics. In [15], the authors have performed a detailed analysis of the influence of photo- and thermo-refractive fluctuations on the noise performance of WGM resonators, and they have found that in $\mathrm{MgF}_{2}$, there are optimal temperatures for which these fluctuations are strongly inhibited. This is one of the properties that strongly motivate our interest for this material in view of microwave photonics applications, where noise is a critical parameter. To the best of our knowledge, the typical $Q$-factor of $\mathrm{MgF}_{2}$ resonators is not available in the literature, even though they have already been fabricated in the past [16]. One of our main objectives is, therefore, to provide this quantitative evaluation in order to facilitate comparison with state-of-the-art $\mathrm{CaF}_{2}$ and fused silica WGM resonators. 
TABLE I

COMPARATIVE PROPERTIES OF VARIOUS MATERIALS USED FOR MANUFACTURING WGM RESONATORS

\begin{tabular}{|l|c|c|c|c|}
\hline \hline Material & Fus. Si & Quartz & $\mathbf{C a F}_{2}$ & $\mathbf{M g F}_{2}$ \\
\hline $\begin{array}{l}\text { Crystal } \\
\text { class }\end{array}$ & $\begin{array}{c}\text { Noncrys- } \\
\text { talline }\end{array}$ & $\begin{array}{c}\text { Hexa- } \\
\text { gonal }\end{array}$ & Cubic & $\begin{array}{c}\text { Tetra- } \\
\text { gonal }\end{array}$ \\
\hline $\begin{array}{l}\text { Transparency } \\
\text { window }(\mu \mathrm{m})\end{array}$ & $0.18-2.5$ & $0.19-2.9$ & $0.2-9$ & $0.12-8.5$ \\
\hline $\begin{array}{l}\text { Refract. index } \\
\text { at } 1550 \mathrm{~nm}\end{array}$ & $n=1.44$ & $\begin{array}{c}n_{o}=1.54 \\
n_{e}=1.53\end{array}$ & $n=1.42$ & $\begin{array}{c}n_{o}=1.37 \\
n_{e}=1.38\end{array}$ \\
\hline $\begin{array}{l}\text { Hardness } \\
\text { (Mohs) }\end{array}$ & $6-7$ & 7 & 4 & 6 \\
\hline $\begin{array}{l}\text { Resistance to } \\
\text { mech. shocks }\end{array}$ & Good & Good & Bad & Good \\
\hline $\begin{array}{l}\text { Resistance to } \\
\mathrm{H}_{2} \mathrm{O} \text { pollution }\end{array}$ & Low & Low & High & High \\
\hline
\end{tabular}

A special equipment was developed for manufacturing the $\mathrm{MgF}_{2}$ disk-resonator. We used a precision adapter and a dedicated polishing machine affording a 200-nm eccentricity. The whole system is hold on an air-bearing support in order to prevent the mechanical influence of vibrations. The polishing process is started from an initial $\mathrm{MgF}_{2}$ bulk-disk with a diameter of $6 \mathrm{~mm}$ and a thickness around $500 \mu \mathrm{m}$. A hole is drilled in the center to allow an easy positioning, and special care should be taken in order to avoid eccentricity problems during the polishing procedure. The rim of the disk has to be reduced to less than $50 \mu \mathrm{m}$ in order to optimize the selectivity of the fundamental WGMs. This thin rim is obtained with two $20^{\circ}$ angle bevels, performed at the edge of the resonator. It is necessary to perform carefully this step with appropriated grindstone and disk speed in order to avoid any splinter that can enlarge dramatically the edge and allow the excitation of higher order WGMs.

After preshaping, manual polishing is then realized carefully with low speed. We need a very good optical quality with very low and regular roughness all around the surface of the disk periphery. Powders with decreasing grain size are used, as for example colloidal silica, cerium oxide, and alumina oxide. Their dilution has to be controlled very precisely. The characterization of the resonator external surface is performed by measuring Newton interference fringes. Using a white light phase shifting interferometer, the rugosity of the rim surface has been evaluated. The result of this measurement is presented in Fig. 1, which displays the surface rugosity of our resonator along two nonparalel axes (perpendicular in our case), over $60 \mu \mathrm{m}$. This double measurement aims to verify that the surface profile is statistically flat, independently of the direction (that is, locally free from scratches). The rugosity is in the range of $1-5 \mathrm{~nm}$, corresponding to 3-12 atoms. Unfortunately, the available instrument permits only the measurement of a small area, while a smooth defect-free surface is required in the whole equatorial region of the resonator.

There are several method to couple the disk-resonator to an optical fiber, such as the prism coupling, the angle-shaped fiber coupling, and the tapered fiber coupling. We have chosen to use

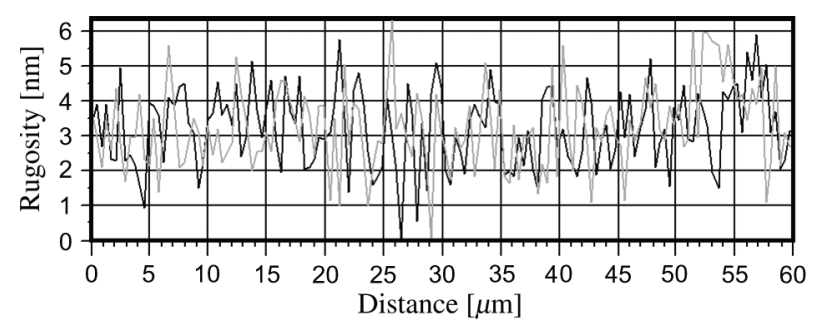

Fig. 1. Experimental characterization of the surface rugosity of our $\mathrm{MgF}_{2}$ resonator using a white light phase shifting interferometer. The rugosity is of the order of few nanometers, and it is measured over $60 \mu \mathrm{m}$ along two perpendicular axis (black and gray lines).

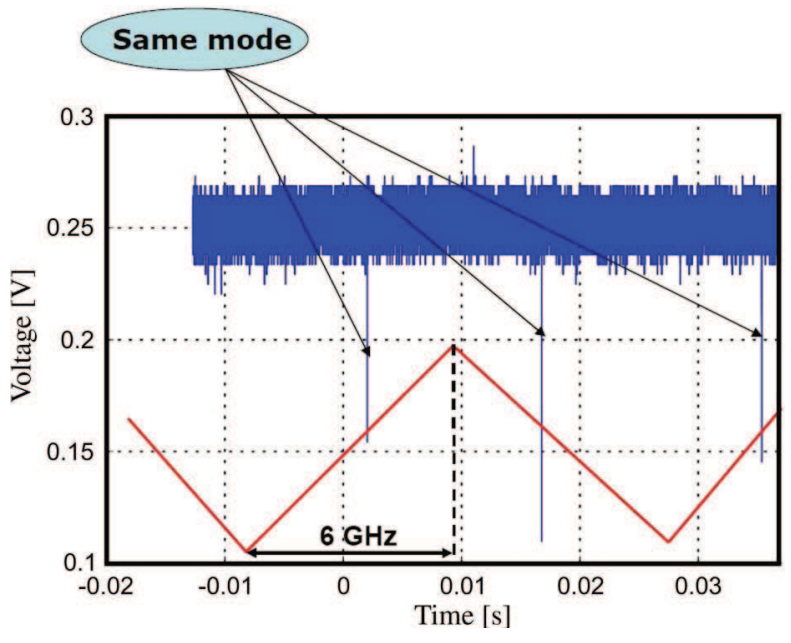

Fig. 2. Detection of a WGM resonance peak near $1550 \mathrm{~nm}$. The same WGM is neatly detected as the laser wavelength is swept back and forth.

the latter because it is generally easier to implement in a laboratory. In this case, the taper is glued on a holder. The holder alloy and geometry match the thermal expansion of the resonator. The taper waist is inferior to $3 \mu \mathrm{m}$. The tapered fiber is set on a three-axis nano-positioning system with a $10-$ to $20-\mathrm{nm}$ resolution.

For measuring the resonance [17], we use the signal from a $1550-\mathrm{nm}$ tunable laser diode powered at $3 \mathrm{~mW}$. The $50-\mathrm{pm}$ wavelength sweep corresponds to $6 \mathrm{GHz}$ in the spectrum. A fast digital real time Lecroy oscilloscope (8600A-type) permits the analysis of the very sharp absorption lines at WGM resonances. It is necessary to use a high-speed resolution oscilloscope for the analysis of these peaks. The oscilloscope is inserted after the photodiode that detects an optical signal coming from the disk resonator, and the resonance peak detection is in the single-mode excitation. The small taper size selects a very thin excitation area, and the resonance measurement setup is in an open loop configuration. Although wavelength span is too small to scan a full free-spectral range (FSR) with a scan rate of $50 \mathrm{~Hz}$, it is still possible to measure the $Q$-factor with the self-homodyne method [18]. A polarization controller is also needed, since the WGM resonances are polarization-dependent. In Fig. 2, we represent the detection of the resonance peak. As written previously, the curve clearly shows the 50-pm wavelength sweep corresponding to $6 \mathrm{GHz}$ in the spectrum, so that the same mode appears regularly. 


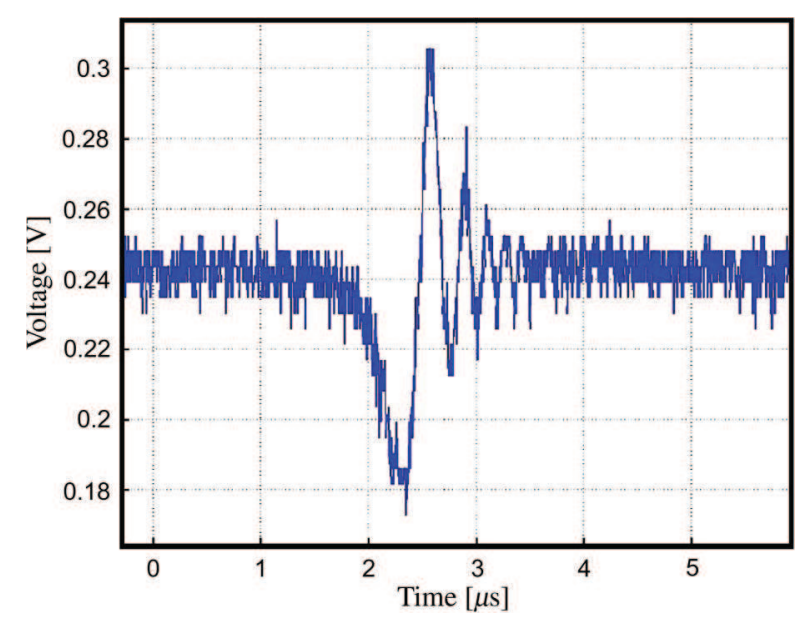

Fig. 3. Cavity ring-down measurement to deduce the $Q$-factor of the $\mathrm{MgF}_{2}$ cavity. The exponential decay time is evaluated to $\tau=0.56 \mu \mathrm{s}$, yielding a $Q$-factor $Q=\omega_{0} \tau / 2=3.4 \times 10^{8}$ at $\lambda_{0}=1550 \mathrm{~nm}$.

The $Q$-factor measurement is deduced from Fig. 3 using the cavity ring-down method. Despite the fact that the resolution is not optimal with the $400-\mathrm{Hz}$ scan rate, curve with voltage (in volts) versus time (in microseconds) gives enough information for the determination of $Q$. The $Q$-factor is extracted from the time delay of the interference path between two beams. The first beam comes from the laser and the second comes from the cavity. Both beams have different wavelengths, and damped oscillations can be observed during cavity unloading. That is why exponentially decaying oscillations are observed on Fig. 3 . The $Q$-factor can be deduced as $Q=\omega_{0} \tau / 2$, where $\omega_{0}=2 \pi c / \lambda_{0}$ is the angular frequency of the laser light, $\lambda_{0}$ is the related wavelength, $c$ is the velocity of light in vacuum, and $\tau$ is the exponential decay time of the electric field (equivalent to twice the photon lifetime). This evaluation of the $Q$-factor is strongly dependent on coupling conditions and the computed value is different according to the way the resonator is coupled to the optical fiber. It can indeed be decomposed in two terms according to $Q^{-1}=Q_{\text {in }}^{-1}+Q_{\text {ext }}^{-1}$, where $Q_{\text {in }}$ is an intrinsic $Q$-factor, while $Q_{\text {ext }}$ is the extrinsic contribution originating from the coupling. Therefore, in order to measure the $Q$-factor with the highest precision, $Q$ must be as close as possible to the intrinsic $Q_{\text {in }}$ which is only related to resonator's properties. This explains why the taper has to be under-coupled. However, under-coupling is not so easy to achieve practically because the tapered fiber has the tendency to stick to the resonator surface, thereby leading to over-coupling. After a careful set of measurements, the cavity ring-down method has provided the value $Q=3.4 \times 10^{8}$ for our $\mathrm{MgF}_{2}$ optical resonator at $1550 \mathrm{~nm}$.

In conclusion, we have fabricated a $\mathrm{MgF}_{2}$ WGM disk-resonator with an high $Q$-factor. Despite its numerous advantages, this crystal is still an unusual bulk material for WGM resonators. The $Q$-factor for this material can in principle be improved by at least one order of magnitude if the fabrication protocol is optimized. In the case of a successful outcome, $\mathrm{MgF}_{2}$ WGM resonators would therefore provide an extremely high $Q$ value that would be stable over a significant lifetime. Such resonators would also be particularly interesting in other applications where the birefringence of the $\mathrm{MgF}_{2}$ crystal is specifically a positive feature.

\section{REFERENCES}

[1] A. B. Matsko, A. A. Savchenkov, D. Strekalov, V. S. Ilchenko, and L. Maleki, "Review of applications of whispering-gallery mode resonators in photonics and nonlinear optics," IPN Progress Report, vol. 42-162, pp. 1-51, 2005.

[2] A. A. Savchenkov, V. S. Ilchenko, A. B. Matsko, and L. Maleki, "Highorder tunable filters based on a chain of coupled crystalline whispering gallery-mode resonators," IEEE Photon. Technol. Lett., vol. 17, no. 1, pp. 136-138, Jan. 2005.

[3] A. A. Savchenkov, W. Liang, A. B. Matsko, V. S. Ilchenko, D. Seidel, and L. Maleki, "Narrowband tunable photonic notch filter," Opt. Lett., vol. 34, pp. 1318-1320, 2009.

[4] A. B. Matsko, L. Maleki, A. A. Savchenkov, and V. S. Illchenko, "Whispering gallery mode based optoelectronic microwave oscillator," J. Mod. Opt., vol. 50, pp. 2523-2542, 2003.

[5] P. Del'Haye, A. Schliesser, O. Arcizet, T. Wilken, R. Holzwarth, and T. J. Kippenberg, "Optical frequency comb generation from a monolithic microresonator," Nature, vol. 450, pp. 1214-1217, 2007.

[6] Y. K. Chembo, D. V. Strekalov, and N. Yu, "Spectrum and dynamics of optical frequency combs generated with monolithic whispering gallery mode resonators," Phys. Rev. Lett., vol. 104, pp. 103902-1-103902-4, 2010.

[7] J. M. Ward, P. Féron, and S. N. Chormaic, "A taper-fused microspherical laser source," IEEE Photon. Technol. Lett., vol. 20, no. 6, pp. 392-394, Mar. 15, 2008.

[8] S. M. Spillane, T. J. Kippenberg, and K. J. Vahala, "Ultra-low threshold Raman laser using a spherical microcavity," Nature, vol. 415, pp. 621-623, 2002.

[9] I. S. Grudinin, A. B. Matsko, and L. Maleki, "Brillouin lasing with a $\mathrm{CaF}_{2}$ whispering gallery mode resonator," Phys. Rev. Lett., vol. 102, pp. 043902-1-043902-4, 2009.

[10] M. L. Gorodetsky, A. A. Savchenkov, and V. S. Ilchenko, "Ultimate $Q$ of optical microsphere resonators," Opt. Lett., vol. 21, pp. 453-455, 1996.

[11] V. S. Ilchenko, A. A. Savchenkov, J. Byrd, I. Solomatine, A. B. Matsko, D. Seidel, and L. Maleki, "Crystal quartz optical whispering-gallery resonators," Opt. Lett., vol. 33, pp. 1569-1571, 2008.

[12] I. S. Grudinin, V. S. Ilchenko, and L. Maleki, "Ultrahigh optical $Q$ factors of crystalline resonators in the linear regime," Phys. Rev. A, vol. 74, pp. 063806-1-063806-9, 2006.

[13] Corning Inc., Magnesium Fluoride Physical and Chemical Properties 2003.

[14] Saint-Gobain Crystals, Magnesium Fluoride 2004.

[15] A. A. Savchenkov, A. B. Matsko, V. S. Ilchenko, N. Yu, and L. Maleki, "Whispering-gallery-mode resonators as frequency references. II. Stabilization,” J. Opt. Soc. Amer. B, vol. 24, no. 12, pp. 2988-2997, 2007.

[16] I. S. Grudinin, A. B. Matsko, A. A. Savchenkov, D. Strekalov, V. S. Ilchenko, and L. Maleki, "Ultra-high $Q$ crystalline microcavities," Opt. Commun., vol. 265, pp. 33-38, 2006.

[17] H. Tavernier, N. N. T. Kim, P. Feron, R. Bendoula, P. Salzenstein, E. Rubiola, and L. Larger, "Optical disk resonators with micro-wave free spectral range for optoelectronic oscillator," in Proc. 22nd Eur. Time and Frequency Forum, Toulouse, France, 2008, Paper FPE-0179.

[18] J. Poirson, F. Bretenaker, M. Vallet, and A. Le Floch, "Analytical and experimental study of ringing effects in a Fabry-Perot cavity. Application to the measurement of high finesses," J. Opt. Soc. Amer. B, vol. 14, pp. 2811-2817, 1997. 